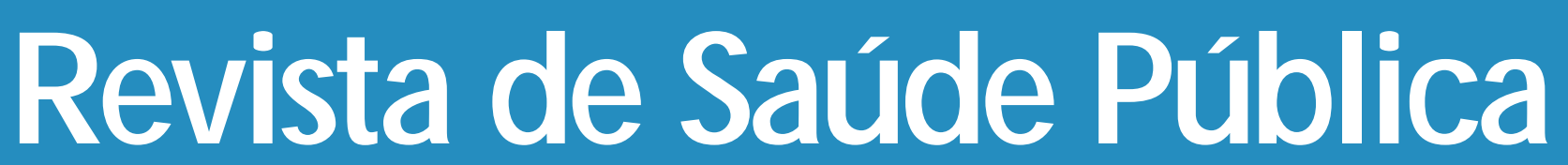

$\begin{array}{llllllll}\mathbf{J} & \mathbf{O} & \mathbf{U} & \mathbf{R} & \mathbf{N} & \mathbf{A} & \mathbf{L}\end{array}$

$0 F$

$\mathbf{P} \mathbf{U}$

B L I C

H E A L T

\title{
Fatores associados à obesidade em adolescentes*
}

\section{Factors associated with obesity among adolescents}

\section{Vania de Matos Fonseca, Rosely Sichieri e G lória Valéria da Veiga}

Departamento de Pediatria do Instituto Fernandes Figueira. Rio de Janeiro, RJ - Brasil (V. M. F.); Instituto de M edicina Social da Universidade Estadual do Rio de Janeiro. Rio de Janeiro, RJ - Brasil (R.S.); Faculdade de N utrição da Universidade Federal Fluminense. Niterói, RJ- Brasil (G.V.V.)

FO NSECA Vania de Matos, Rosely Sichieri e G lória Valéria da Veiga Fatores associados à obesidade em adolescentes* Rev. Saúde Pública, 32 (6): 541-9, 1998

(c) Copyright Faculdade de Saúde Pública da USP. Proibida a reprodução mesmo que parcial sem a devida autorização do Editor Científico. Proibida a utilização de matérias para fins comerciais. All rights reserved. 


\title{
Fatores associados à obesidade em adolescentes*
}

\section{Factors associated with obesity among adolescents}

\author{
Vania de Matos Fonseca, Rosely Sichieri e G lória Valéria da Veiga \\ Departamento de Pediatria do Instituto Fernandes Figueira. Rio de Janeiro, RJ - Brasil (V. M. F.); \\ Instituto de Medicina Social da Universidade Estadual do Rio de Janeiro. Rio de Janeiro, RJ - Brasil \\ (R.S.); Faculdade de N utrição da Universidade Federal Fluminense. Niterói, RJ-Brasil (G.V.V.)
}

\begin{abstract}
Resumo
Introdução

A obesidade na adolescência é um fator preditivo da obesidade no adulto. Assim, foram avaliados os fatores associados à obesidade e o uso do índice de massa morporal (IMC).

Método

Foram avaliados 391 estudantes aferindo-se: consumo de alimentos, hábitos alimentares, características antropométricas dos pais e atividade física. O IMC foi a variável dependente utilizada na regressão linear multivariada.

Resultados

A prevalência de sobrepeso foi $23,9 \%$ para meninos e $7,2 \%$ para meninas. Fazer dieta para emagrecer foi 7 vezes mais freqüente entre meninas do que entre meninos com sobrepeso. Nos meninos, idade, uso de dieta, omissão de desjejum, horas de televisão/"vídeo-game" e obesidade familiar apresentaram associação positiva e significante com IMC. Nas meninas, associaram-se positivamente: uso de dieta, omissão de desjejum e obesidade familiar e negativamente idade da menarca. A correlação do IMC com medidas antropométricas foi maior que 0,7 .
\end{abstract}

Conclusões Um padrão estético de magreza parece predominar entre meninas e elas o atingem com hábitos e consumo alimentar inadequados.

Obesidade. Índice de massa corporal. Estado nutricional

\section{Abstract}

Introduction

Obesity during adolescence is considered a strong predictor of adult obesity. The present study assessed the overweight/obesity prevalence and associated factors in middle class adolescents of a school in the city of Niteroi, Rio de Janeir, Brazil, and evaluated the correlation between body mass index with anthropometric measures of fatness.

Method The analysis covered 391 students aged from 15 to 17 years. Adolescents had their weight, height, skinfold thickness and upper arm circumference measured at school. A food frequency questionnaire (list of 79 items) and a questionnaire including food habits, parents anthropometric characteristics, physical activity and other factors associated with obesity were filled out by the adolescents at school.

*Pesquisa financiada pelo Conselho Nacional de Desenvolvimento Científico e Tecnológico/CNPq (Processo n $\left.{ }^{\circ} 52.0701 / 94-1\right)$.

Correspondência para/Correspondence to: Rosely Sichieri - Rua São Francisco Xavier, 524, $7^{\circ}$ andar - Maracanã - 20531-030 Rio de Janeiro, RJ

- Brasil. E-mail: sichieri@uerj.br.

Recebido em 27.10.1997. Reapresentado em 24.3.1998. Aprovado em 16.4.1998. 
Results The proportion of overweight individuals among boys (Body Mass Index (BMI) greater than the 90th percentile of the Brazilian population) was $23.9 \%$, whereas the prevalence among girls was $7.2 \%$. The fact of being on a slimming diet was a relevant factor for the prediction of BMI and was 7 times more frequent among girls than among boys. Among boys, being on a diet, absence of breakfast, and family body appearance were positively associated with BMI. Among girls these variables were also significantly associated, whereas age at menarche was negatively associated with BMI. Hours of watching TV/videol video-game was associated with BMI only among boys. The correlation coefficient between BMI and measures of fatness varied from 0.7 to 0.9 for boys, and from 0.8 to 0.90 for ginls.

Conclusions BMI appears to be a good indicator of obesity among adolescents and showed that girls demonstrated an exaggerated preoccupation with body image and stereotyped slim body patterns.

Obsesity. Body mass index. Nutritional status.

\section{INTRO DUÇÃO}

Os países desenvolvidos têm concentrado seus esforços, na área de saúde pública, na prevenção das doenças não transmissíveis. Para tanto, ênfase tem sido dada à redução da obesidade, modificação do padrão alimentar e redução do sedentarismo ${ }^{20}$. Os resultados obtidos em relação à redução da obesidade são, contudo desencorajadores, dado que a prevalência de sobrepeso e obesidade, em países como os Estados Unidos, tem sido crescente.

Quanto mais prevalente se torna a obesidade maior o estímulo para se estudar grupos populacionais mais vulneráveis ao problema, onde se possa levantar hipóteses relacionadas à determinação dessa patologia. Um desses grupos é o de adolescentes que, quando obeso, apresenta maior probabilidade de se tornar um adulto obeso ${ }^{18}$.

Diferenças no estado nutricional podem ser decorrentes tanto de influência genética, quanto do meio ambiente, e da interação entre ambos ${ }^{9}$. A correlação entre sobrepeso dos pais e de filhos é grande e decorre do compartilhamento da hereditariedade e a do meio-ambiente.

No Brasil, a PNSN (Pesquisa Nacional de Saúde e Nutrição), de 1989, mostrou que nos adolescentes a prevalência de sobrepeso é importante, sendo que meninas moradoras na região Sul do País e com renda familiar "per capita" acima de 2,2 salários-mínimos apresentavam índice de massa corporal (IMC) maior do que as meninas americanas avaliadas pelo NHANES ("Second National Health and Nutrition Examination Survey") ${ }^{31}$.

A atividade física é um importante determinante das características físicas do adolescente. Dietz ${ }^{4}$ sustenta que a obesidade em adolescentes resulta do desequilíbrio entre atividade reduzida e excesso de consumo de alimentos densamente calóricos, tendo mostrado que o número de horas que um adolescente passa assistindo TV é um importante fator associado à obesidade, acarretando um aumento de $2 \%$ na prevalência da obesidade para cada hora adicional de televisão em jovens de 12 a 17 anos $^{6}$.

O hábito de omitir refeições, especialmente o desjejum, juntamente com o consumo de refeições rápidas, fazem parte do estilo de vida dos adolescentes, sendo considerados comportamentos importantes que podem contribuir para o desenvolvimento da obesidade $^{36}$. O consumo alimentar como um todo não tem sido consistentemente associado ao estado nutricional $^{13}$.

A maturação sexual constitui outro aspecto importante na avaliação do crescimento e desenvolvimento físico dos adolescentes, relacionando-se com o aumento de estatura e peso, desenvolvimento muscular $^{2}$ e aumento dos depósitos de gordura em meninas ${ }^{7}$. Alguns autores têm observado uma relação entre maturação sexual precoce e obesidade em meni$\operatorname{nas}^{12,37}$.

O IMC, embora apresente em adolescentes importante variação com a idade e com a maturidade sexual, tem sido considerado como bom indicador de obesidade em adolescentes ${ }^{15}$, apresentando importante correlação com medidas de dobras cutâneas e com a densitometria ${ }^{26}$. No presente estudo foi adotado o termo sobrepeso para referir tanto a sobrepeso como ao excesso de gordura corporal, assim como tem sido feito por outros autores que definiram em conjunto "sobrepeso e obesidade" em adolescentes com base no IMC'15.

O propósito do presente estudo foi de avaliar: 1) se, à semelhança do descrito em outros países, a avaliação nutricional pelo IMC correlaciona-se à gor- 
dura corporal total e subcutânea dos adolescentes; e 2) verificar os possíveis fatores associados ao estado nutricional avaliado pelo IMC.

\section{MÉTO DO}

Trata-se de um estudo transversal, de amostragem completa, com avaliação dos casos prevalentes de sobrepeso entre estudantes de 15 a 17 anos matriculados numa das escola privadas no Município de Niterói, Estado do Rio de Janeiro, freqüentada por adolescentes da classe média. Dentre as escolas com este perfil, a escolhida foi identificada como a que oferecia melhor acesso ao trabalho a ser desenvolvido.

Participaram do estudo 391 alunos, sendo 183 meninos e 208 meninas. Todos os alunos foram convocados, porém foram registradas 33 perdas decorrentes de faltas; destas, $6(1,4 \%)$ foi devido à recusa. A coleta de dados consistiu na tomada das medidas de peso, estatura, dobras cutâneas (tríceps, subescapular e supra-ilíaca) e perímetro braquial, e na aplicação de um questionário de autoresposta composto de: identificação, quantidade e freqüência de consumo usual de alimentos (lista com 79 alimentos), hábitos alimentares, características antropométricas familiares e atividade física. O questionário foi pré-testado em adolescentes de outra escola de classe média. As medidas antropométricas foram tomadas por um único pesquisador após padronização.

O peso foi medido utilizando-se balança eletrônica, com os alunos sem calçados, usando roupas leves e sem portar objetos pesados. Para a aferição da estatura, uma fita métrica plástica foi afixada na parede sem rodapé, e a estatura medida com os estudantes de meias ou descalços com auxílio de esquadro de madeira. Foram realizadas três mensurações de cada aluno, tomando-se a média das medidas.

Para determinação da prevalência de sobrepeso foi utilizado o referencial do percentil 90 do IMC proposto para população brasileira ${ }^{30}$, com base nos dados da PNSN. As dobras cutâneas foram obtidas utilizando-se um plicômetro da marca Cescorf, pressão constante, tomadas segundo padronização proposta ${ }^{8}$. Os adolescentes foram avaliados de acordo com a localização em curva de percentis das dobra triciptal e dobra subescapular, segundo Shils e Young ${ }^{28}$, os quais utilizaram como referência os dados das crianças americanas brancas e negras a partir do "National Center for Health Statistics", e utilizando o percentil 85 como ponto de corte para obesidade.

O perímetro braquial e a dobra triciptal foram utilizados para o cálculo da área superior do braço(A), área muscular superior do braço $(\mathrm{M})$ e área de gordura superior do braço $(\mathrm{F})^{10}$. Os valores para o perímetro braquial foram avaliados utilizado-se como ponto de corte o percentil 90 como indicador de obesidade, a partir dos dados de crianças americanas ${ }^{1}$.

A avaliação da dieta foi feita através de questionário semi-quantitativo de freqüência de consumo alimentar, previamente testado para adultos em uma população de funcionários do Rio de Janeiro ${ }^{29}$. O questionário, desenhado para dieta brasileira, mostrou correlações com repetidos recordatórios de 24 horas, também aplicados pelos autores, similares àquelas obtidas em outros estudos de validação de questionários de freqüência de alimentos. Para sua utilização entre adolescentes este questionário foi pré-testado em 15 escolares, e, em decorrência, foram adicionados alimentos do tipo hambúrgueres e batata-frita, e foram modificadas porções de alguns alimentos para que se adotasse porções mais usuais para esta faixa etária.

O cálculo da ingestão calórica e composição da dieta em energia, carboidratos, e gorduras foi feito por um programa desenvolvido em SAS ("Statistical Analysis System") $)^{34}$. A composição dos alimentos baseou-se no Programa de Apoio à Nutrição, desenvolvido pela Escola Paulista de Medicina com base na tabela americana de composição química dos alimentos ("handbook, number" $8)^{25}$, na tabela de composição dos alimentos utilizada pelo Estudo Nacional de Despesa Familiar (ENDEF) ${ }^{11}$ e da publicação de Pennington, em 1989, para as preparações não contempladas nas tabelas anteriores ${ }^{24}$.

A atividade física foi avaliada através de questões que englobavam atividades de deslocamento para a escola, recreação (especialmente assistir televisão e jogar "videogame") e esportes, quantificando o tipo, a duração e a freqüência das atividades.

As atividades físicas foram agrupadas em três categorias por grupos de atividades de acordo com a intensidade, aqui chamadas de atividade 1,2 e 3 , o que corresponderia a classificação leve, moderada e intensa, segundo o "Recommended Dietary Allowances"21. Foram registrados o tempo e freqüência dispensados a cada atividade.

Foram, ainda, levantados dados relativos ao estado nutricional dos pais, através de questão que incluía as seguintes categorias em relação ao pai e a mãe: muito gordo, gordo, muito magro, magro e normal. Estes resultados foram correlacionados ao IMC dos adolescentes, após serem agrupados nas seguintes categorias: $0,1 \mathrm{e} 2$, respectivamente para nenhum dos pais gordos; um dos pais gordo; ou os dois pais gordos.

Para avaliação do grau de maturação sexual dos adolescentes foi utilizada a idade da menarca para as meninas e pelos axilares presentes ou ausentes para os meninos. A menarca tem sido usualmente utilizada nos estudos de maturação sexual em meninas, estando relacionada à fase final da maturação sexual ${ }^{2}$. No caso dos meninos, o surgimento dos pêlos axilares ocorre logo após o pico de velocidade em estatura e precede o estágio final de maturação sexual ${ }^{7}$.

Os procedimentos estatísticos utilizados no estudo foram: - para as diferenças entre as médias das variáveis contínuas foi realizado o teste " $t$ "de Student ou análise de variância. Para as variáveis categóricas utilizou-se o teste qui-quadrado ou o exato de Fisher. A análise multivariada através de regressão linear foi escolhida, dado que o IMC não apresenta, para adolescentes, um ponto de corte que 
defina claramente o sobrepeso. Todos estes procedimentos foram realizados com base no SAS, $1985^{34}$.

Todas as variáveis consideradas como possivelmente associadas ao sobrepeso em adolescentes: sexo, idade, maturação sexual, atividade física, horas assistindo televisão ou jogando "video game", ingestão alimentar, freqüência às refeições, fazer dieta para emagrecer e ser descendente de pais gordos, foram analisadas individualmente em relação ao estado nutricional. As variáveis com associação significante $(\mathrm{p}<=0,05)$ foram incluídas na análise de regressão linear multivariada, com o IMC como variável dependente, pois os pontos de corte para o IMC em adolescentes são meramente estatísticos, sem correlação com desenlaces biológicos. Todas as análises utilizaram o software $\mathrm{SAS}^{34}$.

\section{RESU LTAD O S}

O IMC apresentou alta correlação com as outras medidas antropométricas (Tabela 1). Embora uma maior proporção de meninas $(21,2 \%)$ apresentasse valores de perímetro braquial acima do percentil 90 da distribuição das crianças americanas $^{9}$, quando comparadas aos meninos $(8,2 \%)$ o percentual daquelas com área de gordura do braço acima deste percentil $(8,1 \%)$ foi inferior ao dos meninos (12,5\%) (Tabela 2 ). A proporção de meninos classificados com sobrepeso foi superior à apresentada pelas meninas. Os meninos também apresentaram maior prevalência de obesidade avaliada pelas dobras triciptal e subescapular (Tabela 2).

A idade média de ocorrência da menarca foi de 12 anos e 3 meses, e a mediana foi de 12 anos. Para as meninas com sobrepeso, a média de idade da menarca foi de 11 anos e 5 meses, e para aquelas classificadas como normais, 12 anos e 4 meses $(p=0,002)$. O coeficiente de correlação de Pearson entre o IMC e a idade de ocorrência da menarca mostrou uma correlação negativa $(r=-$ $0,20)$ e significante $(\mathrm{p}=0,004)$, ou seja, meninas com maiores valores de IMC tiveram menarcas mais precoces. Na avaliação do grau de maturação sexual dos meninos, apenas um, cuja idade era inferior a 15 anos, não apresentava pêlos axilares.

Em relação à prática de atividade física, os adolescentes com IMC normal e os com sobrepeso se distribuíram de forma bastante próxima. $\mathrm{Ne}$ nhum dos parâmetros avaliados mostrou associação significativa. A maior diferença foi para percentual de meninos normais que não faziam atividade física (Tabela 3 ).

A correlação entre o IMC e a freqüência semanal de atividade para os meninos foi de 0,04 $(p=0,60)$ e para as meninas, $0,17(p=0,01)$. A correlação entre o IMC e o tempo gasto para cada sessão de atividade foi, para o sexo masculino, de $-0,08(\mathrm{p}=0,28)$, e para o sexo feminino, 0,09 $(p=0,18)$. A correlação entre o IMC e o tempo total semanal de atividades no caso dos meninos, foi de $-0,04(\mathrm{p}=0,57)$, e para as meninas, de 0,09 $(\mathrm{p}=0,16)$. A correlação entre IMC e horas diárias de televisão/vídeo/"vídeogame", para os meninos foi de $0,14(\mathrm{p}=0,05)$ e para as meninas $-0,018$ $(p=0,79)$. Este procedimento foi repetido em relação à soma das dobras cutâneas, tudo sido en-

Tabela 1 - Coeficiente de correlação de Pearson entre o índice de massa corporal e outras medidas antropométricas dos adolescentes.

\begin{tabular}{lcc}
\hline Medidas antropométricas & Meninos & Meninas \\
\hline Estatura & 0,04 & $-0,02$ \\
Dobra tríceps & 0,73 & 0,80 \\
Dobra subescapular & 0,78 & 0,80 \\
Dobra supra-ilíaca & 0,82 & 0,77 \\
Soma das dobras & 0,82 & 0,85 \\
Área de gordura braquial & 0,81 & 0,88 \\
Perímetro braquial & 0,91 & 0,90 \\
\hline * Todos os valores de "p" foram iguais ou menores do que 0,0001 , à exceção
\end{tabular}

da estatura cujos $p$-valores foram $>0,60$.

Tabela 2 - Indicadores nutricionais em adolescentes.

\begin{tabular}{|c|c|c|c|c|}
\hline \multirow{2}{*}{$\begin{array}{l}\text { Indicadores } \\
\text { nutricionais } \\
\text { Perímetro braquial acima do percentil 90* }\end{array}$} & \multicolumn{2}{|c|}{$\begin{array}{l}\text { Meninos } \\
(n=183)\end{array}$} & \multicolumn{2}{|c|}{$\begin{array}{l}\text { M eninas } \\
(n=208)\end{array}$} \\
\hline & 15 & $(8,2 \%)$ & 47 & $(21,2 \%)$ \\
\hline Área de gordura do braço acima do perc. 90* & 23 & $(12,5 \%)$ & 18 & $(8,1 \%)$ \\
\hline IMC acima do percentil $90 * *$ & 44 & $(23,9 \%)$ & 16 & $(7,2 \%)$ \\
\hline IMC acima do percentil $85^{* * *}$ & 22 & $(12,1 \%)$ & 16 & $(7,2 \%)$ \\
\hline Dobra tríceps acima do percentil $85^{* * * *}$ & 41 & $(22,3 \%)$ & 30 & $(13,5 \%)$ \\
\hline Dobra subescapular acima do perc. $85^{* * * *}$ & 31 & $(16,8 \%)$ & 26 & $(11,7 \%)$ \\
\hline
\end{tabular}

*Segundo dados da população americana do "N ational Health and N utrition Examination Survey" I (Frizancho ${ }^{10}, 1981$ ). ** Segundo dados para população brasileira da Pesquisa Nacional de Saúde e N utrição (Sichieri e Allam0, 1996). *** Segundo dados para a população americana (Himes e Dietz $\left.{ }^{15}, 1994\right)$. 
contrado uma correlação de $0,18(\mathrm{p}=0,01)$ para os meninos, e $0,02(p=0,76)$ para as meninas.

$\mathrm{O}$ consumo de nutrientes foi maior entre os meninos e meninas normais em comparação com aqueles com sobrepeso. O consumo de energia $(\mathrm{p}=0,04)$ e de carboidratos $(\mathrm{p}=0,02)$ mostraram valores significantemente maiores para os meninos classificados como normais em relação aos com sobrepeso (Tabela 4).

Maior proporção de omissão de desjejum foi referida pelos adolescentes com sobrepeso, 13,6\% dos meninos e 18,7\% das meninas (Figura 1). Observou-se uma diferença significante entre os adolescentes classificados como normais e com sobrepeso, do sexo masculino, em relação à freqüência diária de desjejum ( $\mathrm{p}=0,04$ associado ao teste qui-quadrado). Para as meninas, a diferença não foi significante $(\mathrm{p}=0,52)$, embora tenha sido alto o percentual de meninas que deixavam de fazer o desjejum. A Figura 2 mostra que $9 \%$ dos

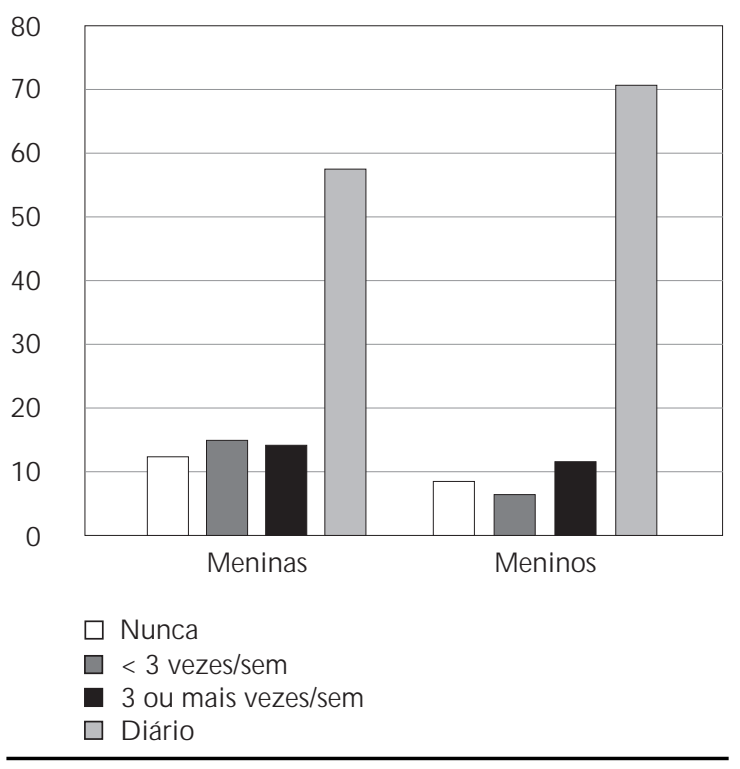

Figura 1 - Freqüência ao desjejum entre adolescentes segundo estado nutricional e sexo.

Tabela 3 - Tempo e freqüência das atividades físicas segundo sexo e o índice de massa corporal dos adolescentes.

\begin{tabular}{|c|c|c|c|c|c|c|c|c|}
\hline \multirow{3}{*}{ Prática de atividade física* } & \multicolumn{4}{|c|}{ Meninos } & \multicolumn{4}{|c|}{ Meninas } \\
\hline & \multicolumn{2}{|c|}{ Normal } & \multicolumn{2}{|c|}{ Sobrepeso } & \multicolumn{2}{|c|}{ Normal } & \multicolumn{2}{|c|}{ Sobrepeso } \\
\hline & & & & & & & & \\
\hline Alguma atividade & 123 & $(87,9 \%)$ & 41 & $(93,2 \%)$ & 160 & $(78,4 \%)$ & 14 & $(87,5 \%)$ \\
\hline \multicolumn{9}{|c|}{ Freqüência semanal de atividade física* } \\
\hline Até 2 vezes & 32 & $(26,2 \%)$ & 9 & $(24,3 \%)$ & 46 & $(28,4 \%)$ & 3 & $(21,4 \%)$ \\
\hline 3 vezes & 26 & $(21,3 \%)$ & 5 & $(13,5 \%)$ & 47 & $(29,0 \%)$ & 2 & $(7,1 \%)$ \\
\hline $4-5$ vezes & 34 & $(27,9 \%)$ & 13 & $(35,1 \%)$ & 48 & $(29,6 \%)$ & 6 & $(42,8 \%)$ \\
\hline Mais de 5 vezes & 30 & $(24,6 \%)$ & 10 & $(24,0 \%)$ & 4 & $(12,9 \%)$ & 4 & $(28,6 \%)$ \\
\hline \multicolumn{9}{|c|}{ Tempo de atividade física por sessão* } \\
\hline $\begin{array}{l}0-45 \text { minutos } \\
46-60 \text { minutos }\end{array}$ & $\begin{array}{l}25 \\
31\end{array}$ & $\begin{array}{l}(21,0 \%) \\
(26,0 \%)\end{array}$ & $\begin{array}{l}11 \\
12\end{array}$ & $\begin{array}{l}(27,5 \%) \\
(30,0 \%)\end{array}$ & $\begin{array}{l}33 \\
60\end{array}$ & $\begin{array}{l}(21,3 \%) \\
(38,7 \%)\end{array}$ & $\begin{array}{l}4 \\
7\end{array}$ & $\begin{array}{l}(28,6 \%) \\
(50,0 \%)\end{array}$ \\
\hline $61-90$ minutos & 20 & $(16,8 \%)$ & 5 & $(12,5 \%)$ & 23 & $(14,8 \%)$ & 1 & $(7,1 \%)$ \\
\hline Mais de 90 minutos & 43 & $(36,1 \%)$ & 12 & $(30,0 \%)$ & 39 & $(25,2 \%)$ & 2 & $(14,3 \%)$ \\
\hline \multicolumn{9}{|c|}{ Tempo total semanal de atividade física* } \\
\hline Até 1 hora & 3 & $(2,6 \%)$ & 1 & $(2,9 \%)$ & 10 & $(6,7 \%)$ & 0 & \\
\hline Entre 1 e 2 horas & 23 & $(20,3 \%)$ & 7 & $(20,6 \%)$ & 24 & $(16,1 \%)$ & 1 & $(7,7 \%)$ \\
\hline $\begin{array}{l}\text { M ais de } 2 \text { horas } \\
\text { Horas diárias TV/vídeo/"vid }\end{array}$ & 87 & $(76,9 \%)$ & 26 & $(76,5 \%)$ & 115 & $(77,2 \%)$ & 12 & $(92,3 \%)$ \\
\hline 0 - 1 hora & 29 & $(22,1 \%)$ & 8 & $(18,6 \%)$ & 45 & $(23,2 \%)$ & 3 & $(21,4 \%)$ \\
\hline Entre 1 e 2 horas & 26 & $(19,8 \%)$ & 10 & $(23,3 \%)$ & 46 & $(23,7 \%)$ & 4 & $(28,6 \%)$ \\
\hline Entre 2 e 3 horas & 30 & $(22,9 \%)$ & 7 & $(16,3 \%)$ & 52 & $(26,2 \%)$ & 3 & $(21,4 \%)$ \\
\hline Mais de 3 horas & 46 & $(35,1 \%)$ & 18 & $(41,9 \%)$ & 51 & $(26,2 \%)$ & 4 & $(28,6 \%)$ \\
\hline
\end{tabular}

*Valores associados ao teste exato de Fisher $>0,40$ tanto para meninos quanto para meninas.

Tabela 4 - Ingestão de nutrientes (média e desvio-padrão) e respectivos P-valores (p) segundo sexo e estado nutricional.

\begin{tabular}{|c|c|c|c|c|c|c|}
\hline \multirow[b]{2}{*}{ N utrientes } & \multicolumn{3}{|c|}{ Meninos } & \multicolumn{3}{|c|}{ Meninas } \\
\hline & $\begin{array}{r}\text { Normal } \\
n=139\end{array}$ & $\begin{array}{c}\text { Sobrepeso } \\
n=44\end{array}$ & $\mathrm{p}^{*}$ & $\begin{array}{c}\text { Normal } \\
n=206\end{array}$ & $\begin{array}{c}\text { Sobrepeso } \\
n=16\end{array}$ & $p^{*}$ \\
\hline Energia (kcal) & $3.188 \pm 1.064$ & $2.819 \pm 965$ & 0,04 & $2.462 \pm 932$ & $2.209 \pm 661$ & 0,28 \\
\hline Gordura (g) & $121 \pm 40$ & $110 \pm 39$ & 0,11 & $97 \pm 39$ & $90 \pm 29$ & 0,48 \\
\hline Carboidrato (g) & $401+149$ & $344 \pm 128$ & 0,02 & $304+125$ & $263 \pm 86$ & 0,09 \\
\hline
\end{tabular}

\footnotetext{
*associado ao teste $\mathbf{t}$ de Student
} 
meninos com sobrepeso fazem dieta, enquanto que, no caso das meninas, este número foi de $62,4 \%$. Mesmo entre as meninas com peso normal, $24,3 \%$ fazem dieta para emagrecer; o que quase não ocorre entre os meninos com IMC normal.

Quanto à aparência física dos pais, as médias de IMC foram mais altas para os que possuíam pais gordos (Tabela 5).

Diferentes modelos de regressão linear multivariada foram construídos utilizando-se como variável dependente o IMC (Tabela 6). Os modelos 1 e 2 não incluem simultaneamente assistir televisão e atividade física, dado que ambas as variáveis referem-se à mesma medida. Incluindo no modelo todas as variáveis significantes na análise bivariada, assistir televisão continuou associada ao IMC para os meninos, a menarca manteve associação e a obesidade familiar também manteve-se associada em ambos os sexos. Foi testada ainda a interação entre fazer dieta para emagrecer e consumo de energia, não sendo encontrado nenhum resultado significante ou mu- dança nos demais coeficientes tanto para meninos quanto para meninas.

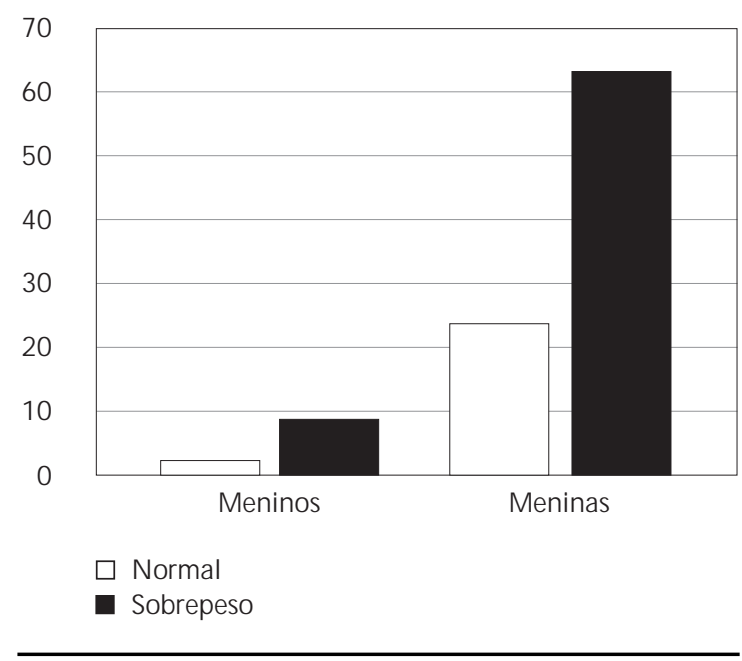

Figura 2 - Estado nutricional e uso de dieta para emagrecer entre os adolescentes segundo sexo.

Tabela 5 - M édias e desvios-padrão (DP) do índice de massa corporal dos adolescentes segundo considerações sobre a aparência física dos pais.

\begin{tabular}{|c|c|c|c|c|}
\hline \multirow{2}{*}{ O pinião } & \multicolumn{2}{|c|}{ Meninos } & \multicolumn{2}{|c|}{ Meninas } \\
\hline & Média & $\mathrm{DP}$ & Média & DP \\
\hline Pai e mãe normais ou magros & $20,8^{*}$ & 3,0 & $20,5^{*}$ & 2,6 \\
\hline Pai ou a mãe gordo ou muito gordo & 22,5 & 3,2 & $21,1^{*}$ & 2,9 \\
\hline Pai e a mãe gordos ou muito gordos & 22,7 & 3,7 & $23,1 *$ & 3,0 \\
\hline
\end{tabular}

Tabela 6 - Modelos lineares de regressão multivariada.

\begin{tabular}{|c|c|c|c|c|c|c|}
\hline \multirow[t]{2}{*}{ Modelo } & \multicolumn{3}{|c|}{ Meninos } & \multicolumn{3}{|c|}{ Meninas } \\
\hline & $\beta$ & p-valor & $\mathrm{R}^{2}$ & $\bar{\beta}$ & p-valor & $\mathrm{R}^{2}$ \\
\hline \multicolumn{7}{|l|}{ Modelo 1} \\
\hline Idade (anos) & 0,74 & 0,001 & 0,07 & 0,11 & 0,57 & 0,04 \\
\hline Energia (100 kcal) & $-0,033$ & 0,14 & & $-0,053$ & 0,01 & \\
\hline Atividade física semanal (horas) & 0,057 & 0,82 & & 0,067 & 0,14 & \\
\hline \multicolumn{7}{|l|}{ Modelo 2} \\
\hline Idade (anos) & 0,56 & 0,001 & 0,08 & 0,11 & 0,55 & 0,02 \\
\hline Energia (100 kcal) & $-0,029$ & 0,20 & & $-0,050$ & 0,02 & \\
\hline Tv/vídeo/"videogame" (horas/dia) & 0,23 & 0,05 & & 0,027 & 0,79 & \\
\hline \multicolumn{7}{|l|}{ Modelo 3} \\
\hline Idade (anos) & 0,41 & 0,001 & 0,17 & 0,10 & 0,60 & 0,20 \\
\hline Energia (100 kcal) & $-0,005$ & 0,83 & & $-0,030$ & 0,20 & \\
\hline Tv/vídeo/"vdeogame" (horas/dia) & 0,27 & 0,02 & & $-0,06$ & 0,55 & \\
\hline Menarca & - & - & - & $-2,74$ & 0,006 & \\
\hline O besidade familiar ( 1 ou os 2 pais) & 1,04 & 0,008 & & 0,94 & 0,001 & \\
\hline Dieta para emagrecer & 4,27 & 0,0016 & & 1,85 & 0,0001 & \\
\hline Desjejum* & 0,16 & 0,52 & & 0,49 & 0,03 & \\
\hline
\end{tabular}




\section{DISCU SSÃO}

Uma tendência secular de aumento da prevalência de obesidade vem sendo referida em estudos populacionais. Sorensen e Price ${ }^{33}$ estudaram o IMC de 38.132 jovens dinamarqueses do sexo masculino e identificaram o início deste aumento nas coortes de nascimento a partir da década de 40 . Gortmaker e col. ${ }^{14}$ analisaram inquéritos americanos (NHES e NHANES) nas décadas de 60, 70 e 80, e mostraram aumento na prevalência de obesidade em crianças e jovens (6 a 17 anos), o mesmo ocorrendo com a população adulta ${ }^{16}$.

No Brasil, esta tendência também tem sido observada na população adulta, haja visto os dados comparativos dos dois inquéritos nacionais ENDEF, em 1975, e PNSN, em 1989, os quais revelaram um aumento da ordem de $56,3 \%$ nos homens e $39,7 \%$ nas mulheres em relação ao sobrepeso; e, 92,0\% nos homens e $69,6 \%$ nas mulheres para a obesidade ${ }^{32}$.

Dietz e col. ${ }^{5}$ discutem os fatores de risco metabólicos e epidemiológicos da obesidade infantil enfatizando que a obesidade é o resultado da interação entre a susceptibilidade do hospedeiro e um meio ambiente que promova a doença. Segundo esses autores, exceto para a associação entre obesidade e horas assistindo televisão, as correlações comportamentais permanecem não especificadas, e o objetivo dos estudos desta década seria a identificação e modificação desses comportamentos.

No presente estudo, nenhuma diferença significante entre os indicadores que compuseram a avaliação da atividade física (freqüência semanal, tempo de cada sessão e tempo total semanal das atividades) foi detectada comparando os meninos normais e aqueles com sobrepeso. Contudo, horas assistindo televisão associou-se positivamente ao IMC, à semelhança do proposto para crianças americanas ${ }^{6}$ . Bradfield e col. ${ }^{1}$ não observaram nenhuma diferença significante entre os registros de 3 dias de atividade física entre obesos e não obesos, todavia seu estudo restringiu-se à meninas. Sunnergardh e $\mathrm{Col}^{35}$, apesar de não encontrarem correlação entre atividade física diária e percentual de gordura corporal, registraram uma tendência em direção a uma mais alta percentagem de gordura corporal em crianças ( 8 a 13 anos) menos ativas do que nas mais ativas. $\mathrm{Ou}-$ tros estudos, porém em adultos, indicaram correlações negativas ${ }^{19,26,27}$ entre IMC e exercícios. No presente estudo, a correlação entre o IMC e a freqüência semanal de atividade física em meninas foi de $0,17(\mathrm{p}=0,01)$ mostrando que as meninas com sobrepeso praticavam atividade física com mais freqüência do que as meninas com peso normal. Esta é uma limitação de estudos transversais como este, uma vez que adolescentes que valorizam "o ser magro" fazem mais atividade física e restringem o consumo de alimentos. Por outro lado, o presente estudo mostra que a baixa prevalência de sobrepeso entre meninas pode ser decorrente, pelo menos em parte, de maior atividade física. Comparando estes dados com o inquérito realizado no Município do Rio de Janeiro observa-se que a prevalência entre os meninos foi próxima à encontrada nesse município, enquanto para as meninas esta prevalência foi de aproximadamente $50 \%{ }^{3}$.

Os resultados da análise multivariada mostraram que para os meninos o IMC aumentou com a idade, o que segundo Obarzanek ${ }^{22}$ seria esperado durante o crescimento. A idade não se associou ao aumento de IMC entre as meninas estudadas, de um lado porque elas se encontram em faixa etária de crescimento reduzido e também porque as adolescentes de Niterói demonstraram uma excessiva preocupação com o manter-se magro. Fazer dieta para emagrecer foi um dos mais importante fatores explicativos para as variações do IMC nas meninas. Para os meninos fazer dieta também foi positivamente e significantemente relacionado ao IMC, apontando maior uso de dietas nos valores mais altos de IMC, embora o percentual de meninos que tenha referido estar em dieta para emagrecer tenha sido muito menor.

A idade da menarca no presente estudo foi também um importante fator preditivo do IMC; estes achados são consistentes com outros autores ${ }^{12,37}$.

Uma variável também associada às variações do IMC foi a descendência de pais gordos. Os maiores valores de IMC foram encontrados para as adolescentes com história de adiposidade de ambos os pais.

Um hábito muito comum entre os adolescentes é omitir refeições. Esta atitude é referida por Tojo ${ }^{36}$ como um fator de risco nutricional para os adolescentes. Nos dados do presente estudo, as meninas omitiram mais refeições que os meninos, sugerindo que mesmo aquelas com pesos normais lançam mão do recurso da omissão de refeições para evitar o ganho de peso. Porém, tanto entre os meninos quanto entre as meninas, omissão do desjejum foi positiva e significantemente associada ao IMC. Em concordância com a omissão de refeições, o consumo de energia relacionou-se inversamente com o IMC, mesmo depois de ser ajustado para atividade física. Uma outra possibilidade de explicação para esta associação negativa é a subestimação de consumo entre os 
mais gordos ${ }^{17}$, principalmente em relação aos meninos. Ortega e col. ${ }^{23}$ observaram em adolescentes obesos subestimação da ingestão de alimentos ricos em açúcar. Outra explicação provável é de que fatores da dieta são mais importantes no início da obesidade, ao passo que atividade física e/ou fatores metabólicos têm importância na manutenção da obesidade ${ }^{17}$.

Em conclusão, a despeito do caráter restrito e transversal do presente estudo, os resultados indicam que o IMC é um indicador de obesidade para adolescentes e apontam a influência familiar e o sedentarismo, particularmente dos meninos, como importante fatores no desenvolvimento do sobrepeso. Um padrão estético de magreza parece predominar entre as meninas e elas o atingem com hábitos e consumo alimentar inadequados.

\section{REFERÊN CIAS}

1. BRADFIELD, R. B. et al. Energy expenditure and heart rate of obese high school girls. Am. J. Clin. Nutr., 24: 1482-8, 1971.

2. COLLI, A. S. Maduración sexual de los adolescentes de São Paulo. In: Organización Panamericana de la Salud. La salud del adolescent y joven en las Americas. Washington, D.C., 1985. p.249-58 (Publicación científica 489).

3. COSTA, R. Associação de medidas antropométricas com a pressão arterial em uma amostra de adolescentes no Município do Rio de Janeiro. Rio de Janeiro, 1997. [Tese de Mestrado - Instituto de Medicina Social da UERJ].

4. DIETZ, W. H. Factors increasing risk of obesity and potencial for prevention overweight in childhood. In: Workshop in Prevention of Obesity Population at Risk, Etiologic Factors and Intervention Strategies, Baltimore, National Institutes of Health/ National Institute of Diabetes and Digestive and Kidney Diseases, 1993. p.64.

5. DIETZ, W.H. et al. Epidemiologic and metabolic risk factors for childhood obesity. Klin. Padiatr., 202: 29-32, 1990.

6. DIETZ, W.H. \& GORTMAKER, S.L. Do we fatten our children at the television set? Obesity and television viewing in children and adolescents. Pediatrics, 75: 807-12, 1985.

7. DUARTE, M.F.S. Maturação física: uma revisão na literatura, com especial atenção à criança brasileira. $\mathrm{Cad}$. Saúde Pública, 9(Supl.1): 71-84, 1993.

8. DURNIN, J.V.G.A. \& RAHAMAN, V.M. The assessment of the amount of fat in the human body from measurements of skinfold thickness. Br. J. Nutr.,21:681-8, 1967.

9. EVELETH, P.B. \& TANNER, J.M. World variation in human growth. 2 ed. Cambridge, Cambridge University Press, 1990

10. FRISANCHO, A.R. News norms of upper limb fat and muscle areas for assessment of nutritional status. Am. J. Clin. Nutr., 34: 2540-5, 1981.

11. FUNDAÇÃO IBGE. Tabela de composição dos alimentos. Rio de Janeiro, 1985. (ENDEF - Estudo Nacional de Despesa Familiar)

12. GARN, S.M. \& HASKELL, B.A. Fat thickness and developmental status in childhood and adolescence. Am. J. Dis. Child., 99:746-51, 1960.
13. GAZZANIGA, J. M. \& BURNS, T.L. Relationship between diet composition and body fatness, with adjustment for resting energy expenditure and physical activity, in preadolescent children. Am. J. Clin. Nutr., 58: 21-8, 1993.

14. GORTMAKER, S. L. et al. Increasing pediatric obesity in United States. Am. J. Dis. Child., 141: 535-40, 1987.

15. HIMES, J. H. \& DIETZ, W. H. Guidelines for overweight in adolescent preventive services: recommendations from an expert committee. Am. J. Clin. Nutr., 59: 307-16, 1994.

16. HUBBARD, V.S. Prevention of obesity: populations at risk, etiologic factors and intervention strategies: introduction. Obes. Res., 3: 75s-6s, 1995.

17. KLESGES, R. C. et al. Accuracy of self-reports of food intake in obese and normal weight individuals: effects of parental obesity on reports of children's dietary intake. Am. J. Clin. Nutr., 48: 1252-6, 1988

18. MALINA, R. M. \& BOUCHARD, C. Growth, maturation and physical activity. New York, Versa Press, 1991.

19. MILLER, W. C. et al. Diet composition, energy intake, and exercise in relation to body fat in men and women. Am. $J$. Clin. Nutr., 52: 426-30, 1990.

20. NATIONAL RESEARCH COUNCIL. Committee on Diet and Health. Diet and health: implications for reducing chronic diseases risk. Washington, DC, Academy Press, 1989. p.99-135, 563-92.

21. NATIONAL RESEARCH COUNCIL. Commission on Life Sciences. Subcommittee on the Tenth Edition of the RDAs. Recommended dietary allowances. Washington, DC, National Academy Press, 1989.

22. OBARZANEK, E. et al. Energy intake and physical activity in relation indexes of body fat: the National Heart, Lung, and Blood Institute Growth and Health Study. Am. J. Clin Nutr., 60: 15-22, 1994.

23. ORTEGA, R. M. et al. Relationship between diet composition and mass index in a group of Spanish adolescents. Brit. J. Nutr., 74: 765-73, 1995.

24. PENNINGTON, J. A. T. Food values of portions used. 15th ed. New York, Perinnial Library- Harper 1989. 
25. PROGRAMA de Apoio à Nutrição. [Disquete]. Versão 2.5 São Paulo, SP, Escola Paulista de Medicina, 1995.

26. REVICKI, D. A. \& ISRAEL, R. G. Relationship between body mass and measure of body adiposity. Am. J. Public Health, 76: 992-4, 1986.

27. ROMIEU, I. et al. Energy intake and other determinants of relative weight. Am. J. Clin. Nutr., 47: 406-12, 1988.

28. SHILS, M.E. \& YOUNG, V. R. Modern nutrition in health and disease. 17th ed. New York (Lea Feibiger), 1988.

29. SICHIERI, R. Gender difference in the association of reported energy consumption with energy requirement in a sample of Brazilian workers. Am. J. Clin. Nutr., 61: 904, 1995.

30. SICHIERI, R. \& ALLAM, V. C. Avaliação do estado nutricional de adolescentes brasileiros através do índice de massa corporal. J. Pediatr. (S. Paulo), 72: 80-4, 1996.

31. SICHIERI, R. et al. High temporal, geographic and income variation in body mass index among adults in Brazil. Am. J. Public Health, 84: 793-8, 1994.
32. SICHIERI, R et al. Growth and body mass index of Brazilians ages 9 through 17 years. Obes. Res., 3: $117 \mathrm{~s}-21 \mathrm{~s}, 1995$.

33. SORENSEN, T. I. \& PRICE, R. A. Secular trends in body mass index among Danish young men. Int. J. Obes., 14: 231-9, 1990.

34. STATISTICAL Analysis Sistem - SAS. [Disquete]. version 6.4. Cary, NC, SAS Institute Inc., 1985

35. SUNNEGARDH, J. et al. Physical activity in relation to energy intake and body fat in 8-and 13-year-old children in Sweden. Acta Paediatr. Scand. 75: 955-63, 1986.

36. TOJO, R. et al. Nutrición en el adolescente. Fatores de riesgo biopsicosociales. An. Españoles Pediatr., 35 (Supll. 46): 74-83, 1991

37. VEIGA, G.V. et al. Adaptação do critério antropométrico para avaliação do estado nutricional de adolescentes em dois níveis socioeconômicos no Município de São Paulo. J. Pediatr. (S. Paulo), 68: 26-33, 1992. 